\title{
Multi-Frequency Broadband Optimization of Spaceborne Reflectarrays for Space Applications
}

\author{
Daniel R. Prado*, Manuel Arrebola ${ }^{\dagger}$, Marcos R. Pino ${ }^{\dagger}$ and George Goussetis* \\ *Institute of Sensors, Signals and Systems, Heriot-Watt University, Edinburgh, U.K. Email: \{dr38, g.goussetis\}@ hw.ac.uk \\ ${ }^{\dagger}$ Group of Signal Theory and Communications, Universidad de Oviedo, Spain. Email: \{arrebola, mpino\}@uniovi.es
}

\begin{abstract}
Direct-to-home (DTH) applications usually require a radiation pattern with a given footprint on the surface of the Earth and impose stringent cross-polarization requirements in the form of crosspolar discrimination (XPD) or crosspolar isolation in a given bandwidth. This paper describes a multifrequency broadband optimization procedure and performance results of a very large spaceborne reflectarray for DTH application in a $10 \%$ bandwidth. The proposed design methodology is based on the generalized intersection approach and the use of a multi-resonant unit cell with multiple degrees of freedom (DoF). The procedure is divided into three stages to facilitate convergence towards a broadband performance. First, a initial narrowband design at central frequency is obtain. Then, a broadband optimization including XPD requirements is carried out with a limited number of DoF. Finally, more DoF are included in the last stage optimization to obtain a broadband reflectarray with improved cross-polarization performance.

Index Terms - reflectarrays, optimization, space communications, shaped-beam, generalized intersection approach
\end{abstract}

\section{INTRODUCTION}

An important drawback of reflectarray antennas is their narrow bandwidth due to the use of resonant elements and the differential spatial delay [1]. The use of sub-wavelength elements, with a periodicity smaller than half a wavelength at the working frequency, improves the bandwidth of reflectarrays [2]. However, these elements have an important limitation in the total range of phase-shift provided, which limits the design of advance reflectarrays with shaped beams. In addition, sub-wavelength elements do not solve the differential spatial delay, which is critical in very large reflectarrays [1]. The latter issue may be solved with the use of true time delay elements [3], although their topology may be complex when dealing with two polarizations at the same time and thus not suitable for a direct optimization of the whole reflectarray antenna.

In this work, an alternative method employing a multiresonant element and performing an optimization at several frequencies is proposed for dual-polarized, contoured-beam reflectarrays with improved cross-polarization performance. In this way, both bandwidth limitations are overcome, since the multi-resonant elements provide more bandwidth [1] and the optimization at several frequencies minimizes the differential spatial delay produced by the planar nature of the reflectarray antenna. A very large reflectarray with southern

This work was supported in part by the Ministerio de Ciencia, Innovación y Universidades under the project TEC2017-86619-R (ARTEINE); by the Ministerio de Economía, Industria y Competitividad under the project TEC2016-75103-C2-1-R (MYRADA); by the Gobierno del Principado de Asturias/FEDER under the project GRUPIN-IDI/2018/000191; by the Gobierno del Principado de Asturias through the Programa "Clarín" de Ayudas Postdoctorales/Marie Curie COFUND under the project ACA17-09; and by Ministerio de Educación, Cultura y Deporte/Programa de Movilidad "Salvador de Madariaga" (Ref. PRX18/00424).

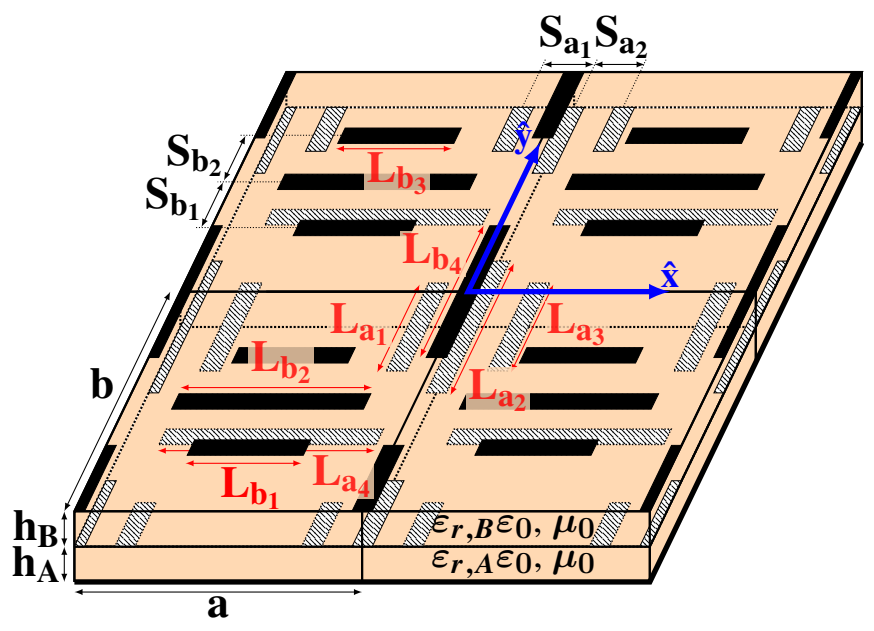

Fig. 1. Multi-resonant unit cell based on two sets of coplanar dipoles in two layers of metallization.

Asia coverage is employed to demonstrate this design strategy, using the generalized intersection approach [4] as the optimization algorithm. In addition, requirements for crosspolarization performance in the form of crosspolar discrimination (XPD) are considered. The optimized reflectarray complies with the copolar specifications in a $10 \%$ bandwidth $(11.80 \mathrm{GHz}-13.20 \mathrm{GHz})$ while the XPD is better than $33 \mathrm{~dB}$ in the whole band.

\section{Design Methodology for BROADBAND PERFORMANCE}

\section{A. Reflectarray analysis}

A single-offset configuration is considered, in which a feed (a horn antenna) illuminates the surface of the reflectarray. The incident tangential field $\vec{E}_{\text {inc }}(f)$ will vary with frequency, and the reflected tangential field is then calculated as:

$$
\vec{E}_{\text {ref }}(f)=\boldsymbol{R}(f) \vec{E}_{\text {inc }}(f),
$$

where

$$
\boldsymbol{R}(f)=\left(\begin{array}{cc}
\rho_{x x}(f) & \rho_{x y}(f) \\
\rho_{y x}(f) & \rho_{y y}(f)
\end{array}\right)
$$

is the matrix of reflection coefficients, which also depends on frequency, as well as on other factors such as the substrate of the unit cell, its geometry, etc. These coefficients are computed with a full-wave analysis tool assuming local periodicity. For the case at hand, the unit cell shown in Fig. 1 is considered, which is analysed by the method of moments based on local periodicity (MoM-LP) described in [5].

After the reflected tangential field in (1) has been obtained, the far field is computed using the first principle of equivalence. The copolar and crosspolar components are then 


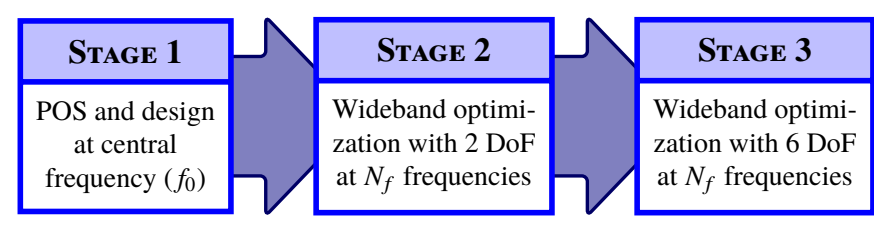

Fig. 2. Flowchart of the broadband optimization procedure.

readily obtained using Ludwig's third definition of crosspolarization. For its use in a broadband optimization procedure, the analysis described here is carried out independently at a number of frequencies within a specified band.

\section{B. Broadband Design Methodology}

The design methodology is divided into several stages to facilitate convergence towards a broadband performance and it is based on the multi-resonant cell shown in Fig. 1. This unit cell is composed of two sets of four parallel dipoles each in two layers of metallization. Each set of four dipoles controls the phase-shift for each linear polarization. In addition, the dipoles introduce different resonances, providing broadband performance [1].

Fig. 2 shows the flowchart of the broadband design procedure. The result of one given stage is the starting point for the next. Thus, if the results after an optimization are not suitable because the algorithm has fallen into an undesired local minimum, only that stage needs to the repeated, accelerating the design process.

The first stage consists of a phase-only synthesis (POS) and a layout design at central frequency. For the POS, a focused beam in a direction $\left(\theta_{0}, \varphi_{0}\right)$ is employed as starting point, whose phase-shift distribution is analytically obtained through:

$$
\phi\left(x_{k}, y_{k}\right)=k_{0}\left(f_{0}\right)\left(d_{k}-\left(x_{k} \cos \varphi_{0}+y_{k} \sin \varphi_{0}\right) \sin \theta_{0}\right),
$$

where $\left(x_{k}, y_{k}\right)$ are the coordinates of the $k$-th reflectarray element; $d_{k}$ is the distance from the feed to the $k$-th element; and $k_{0}\left(f_{0}\right)$ is the wavenumber in vacuum at central frequency $\left(f_{0}\right)$. The phase-shift in (3) corresponds to the phase of the reflection coefficient $\rho_{x x}$ for linear polarization $\mathrm{X}$, or to $\rho_{y y}$ for polarization Y. Since the POS can only impose requirements on the copolar pattern, the resulting reflectarray will meet the copolar specifications, but in general it will not comply with cross-polarization requirements. In addition, it will have narrowband performance.

Thus, in the second stage, a broadband optimization will be carried out, imposing both copolar and cross-polarization requirements. This is an intermediate step in which a limited number of degrees of freedom (DoF) are employed in the optimization. In particular, and based on the unit cell shown in Fig. 1, two DoF per element will be considered, $T_{x}$ and $T_{y}$, defined as follows [6]:

$$
\begin{aligned}
& L_{a_{4}}=T_{x} ; \quad L_{b_{1}}=L_{b_{3}}=0.63 T_{x} ; \quad L_{b_{2}}=0.93 T_{x} \\
& L_{b_{4}}=0.95 T_{y} ; \quad L_{a_{1}}=L_{a_{3}}=0.58 T_{y} ; \quad L_{a_{2}}=T_{y} .
\end{aligned}
$$

The optimization will be carried out at three frequencies, central $(12.50 \mathrm{GHz})$ and extremes $(11.80 \mathrm{GHz}, 13.20 \mathrm{GHz})$, which corresponds to a $10 \%$ bandwidth. This is done by conveniently modifying the cost function of the generalized intersection approach [4], which now takes the form:

$$
\begin{aligned}
F= & \sum_{f=1}^{N_{f}} \sum_{m=1}^{M}\left\{W_{f, 1}\left(\vec{r}_{m}\right)\left[\mathrm{CP}_{\min , f}^{\prime}\left(\vec{r}_{m}\right)-\mathrm{CP}_{\min , f}\left(\vec{r}_{m} ; \bar{\xi}\right)\right]+\right. \\
& \left.W_{f, 2}\left(\vec{r}_{m}\right)\left[\mathrm{XPD}_{\min , f}^{\prime}\left(\vec{r}_{m}\right)-\mathrm{XPD}_{\min , f}\left(\vec{r}_{m} ; \bar{\xi}\right)\right]\right\}^{2}
\end{aligned}
$$

where $N_{f}$ is the number of frequencies at which the optimization is carried out, $N_{f}=3$ in the present case; $M$ is the number of coverage zones; $\vec{r}_{k}=(u, v)_{m}$, with $u=\sin \theta \cos \varphi$ and $v=\sin \theta \sin \varphi$, is an observation point in the coverage zone; $W_{f}$ is a weighting function which depends on the frequency and observation point; $\mathrm{CP}_{\min , f}^{\prime}\left(\vec{r}_{m}\right)$ and $\mathrm{XPD}_{\min , f}^{\prime}\left(\vec{r}_{m}\right)$ are the reference parameters being optimized $\left(\mathrm{CP}_{\min }\right.$ is the minimum gain in a coverage area); $\mathrm{CP}_{\min , f}\left(\vec{r}_{m} ; \bar{\xi}\right)$ and $\mathrm{XPD}_{\min , f}\left(\vec{r}_{m} ; \bar{\xi}\right)$ are the current parameters generated by the reflectarray, which depends on the vector of optimizing variables $\bar{\xi}$. In this case, the vector $\bar{\xi}$ includes the values of $T_{x}$ and $T_{y}$ for all reflectarray elements that are optimized. This cost functions takes into account both copolar and cross-polarization requirements. In addition, the reference parameters are the goals to be achieved by the optimization.

Finally, in the third stage the number of DoF is increased to six per unit cell. The length of all dipoles is free to vary with the exception of the lateral dipoles for each linear polarization, which will be the same to maintain the cell symmetry ( $L_{a_{1}}=L_{a_{3}}$ and $L_{b_{1}}=L_{b_{3}}$, see Fig. 1 ). Thus, for each element, the vector $\bar{\xi}$ will include the lengths $L_{a_{i}}$ and $L_{b_{i}}$, with $i=1,2,4$.

\section{RESULTS FOR A REFLECTARRAY WITH SOUTHERn ASIAN COVERAGE}

\section{A. Antenna Definition and Requirements}

The considered reflectarray is elliptical with axes $1128 \mathrm{~mm} \times 1080 \mathrm{~mm}$ in a single offset configuration. It is comprised of 6640 elements in a regular grid of periodicity $12 \mathrm{~mm}$. The feed is placed at $(-352.9,0.0,1061.7) \mathrm{mm}$ from the reflectarray center and it is modelled as a $\cos ^{q} \theta$ function, in which the parameter $q$ defines the directivity of the feed and varies with frequency. Specifically, the values for $q$ are 16,18 and 20 at $11.80 \mathrm{GHz}, 12.50 \mathrm{GHz}$ and $13.20 \mathrm{GHz}$, generating an illumination taper of $-16.1 \mathrm{~dB},-17.9 \mathrm{~dB}$ and $-19.7 \mathrm{~dB}$, respectively. The width of the dipoles is set to $0.5 \mathrm{~mm}$ while the separation center to center between them is $2.5 \mathrm{~mm}$. Commercial substrates were chosen, the Arlon AD255C for layer A with $h_{A}=2.363 \mathrm{~mm}$ and $\varepsilon_{r, A}=2.17-j 0.0020$, and the Diclad 880 for layer B, with $h_{B}=1.524 \mathrm{~mm}, \varepsilon_{r, B}=2.55-j 0.0023$.

Fig. 3 shows the footprint of the southern Asia coverage considered in this work. It has two areas: zone 1 includes the countries India, Nepal, Bhutan, Bangladesh and Sri Lanka; and zone 2 includes Pakistan and Afghanistan. This footprint corresponds to the coverage of the SES-12 satellite, placed in geostationary orbit at $95^{\circ} \mathrm{E}$. The official specifications [7] stipulate an EIRP of $52 \mathrm{dBW}$ for zone 1 and $48 \mathrm{dBW}$ for zone 2, which can be converted into gain with:

$$
G(\mathrm{dBi})=\operatorname{EIRP}(\mathrm{dBW})-P_{t}(\mathrm{dBW}),
$$

where $P_{t}$ is the power of the transponder. Considering $P_{t}=$ $150 \mathrm{~W}$, it gives a gain requirement of $30 \mathrm{dBi}$ and $26 \mathrm{dBi}$ for 


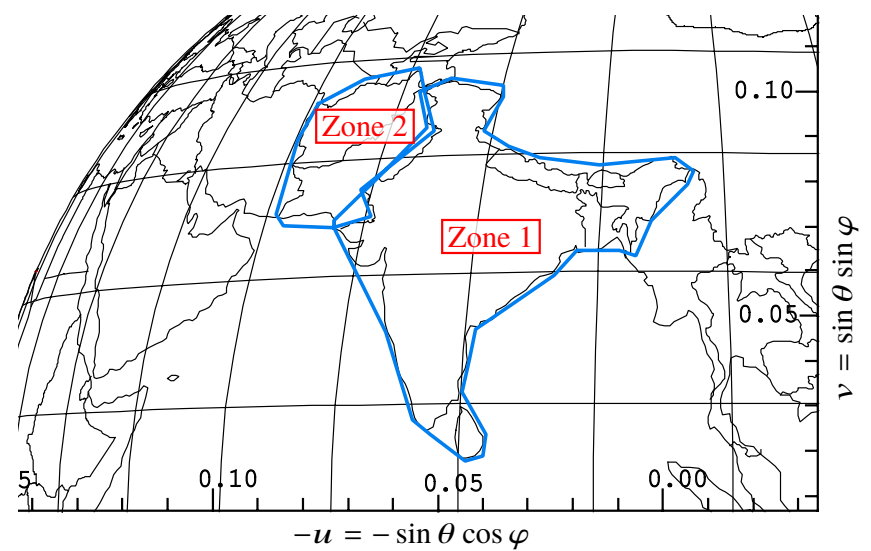

Fig. 3. Footprint of the southern Asian coverage, with $(u, v)$ coordinates in the satellite coordinate system.

zones 1 and 2, respectively. The design process will take into account typical pointing errors $\left(0.1^{\circ}\right.$ in pitch and roll, and $0.5^{\circ}$ in yaw) and it will be carried out in dual-linear polarization, with the same specifications in both polarizations. The crosspolarization goal is to achieve a minimum of $33 \mathrm{~dB}$ for the XPD.

\section{B. Central Frequency Design}

The first step is the design at central frequency of a shapedbeam reflectarray that complies with the requirements. For that purpose, a phase-only synthesis (POS) is carried out using the generalized intersection approach [8]. The result is two phase distributions, one for each linear polarization, such that the generated copolar pattern complies with the specifications. The following step is to obtain a layout by adjusting the element geometry to provide the required phaseshift. This is done with a zero-finding routine, for instance, the Newton-Raphson method. A detailed explanation of this step can be found in [9].

Although the obtained layout complies with the copolar specifications at central frequency $(12.5 \mathrm{GHz})$, it is narrow bandwidth. For instance, the minimum copolar gain for zone 1 at $11.80 \mathrm{GHz}$ is $25.26 \mathrm{dBi}$. Moreover, this reflectarray does not even comply with the cross-polarization requirements at central frequency, since the POS only deals with copolar specifications due to the simplifications in the analysis of the unit cell [8]. Thus, a direct layout optimization employing the MoM-LP tool will be carried out next.

\section{Broadband Optimization Results}

The broadband optimization is carried out with the algorithm presented in [9]. It employs the MoM-LP directly in the optimization loop to perform the unit cell analysis. First, only two DoF per element are considered, with a total of 13097 variables. The goal is to improve the copolar performance of the antenna in a $10 \%$ bandwidth with a limited number of DoF while also imposing cross-polarization requirements. However, at this stage is more important to improve the copolar pattern. Moreover, by improving the copolar pattern, the XPD is improved indirectly due to its definition [9].

The worst case for the minimum copolar gain after stage 1 was zone 1 at $11.80 \mathrm{GHz}$ for polarization $\mathrm{Y}$ with a value of $24.19 \mathrm{dBi}$ (requirement of $30 \mathrm{dBi}$ ), and zone 2 at $13.20 \mathrm{GHz}$ for polarization $\mathrm{Y}$ with a value of $22.56 \mathrm{dBi}$ (requirement of $26 \mathrm{dBi}$ ). After the optimization carried out in stage 2, those values improve to $28.80 \mathrm{dBi}$ and $26.45 \mathrm{dBi}$, respectively. Regarding the cross-polarization performance, the $\mathrm{XPD}_{\text {min }}$ (minimum value of the XPD in a given zone) also improves. The lowest value of $\mathrm{XPD}_{\min }$ in the whole band in stage 1 is $24.40 \mathrm{~dB}$. After the optimization with two DoF per element, the worst value is $27.12 \mathrm{~dB}$. These $\mathrm{XPD}_{\min }$ values correspond to zone 1 at $13.20 \mathrm{GHz}$ for polarization $\mathrm{Y}$.

After the optimization of stage 2, the copolar pattern is close to fulfil requirements. In fact, zone 2 complies with the requirements in the whole band for both polarizations, while zone 1 for polarization $\mathrm{X}$ is very close (worst value is $29.77 \mathrm{dBi}$ ) and polarization $\mathrm{Y}$ presents a worst value of $28.80 \mathrm{dBi}$ at $11.80 \mathrm{GHz}$, but complies at central frequency and is very close at $13.20 \mathrm{GHz}$ with a value of $29.45 \mathrm{dBi}$. However, the cross-polarization performance is far from complying. The worst value of $\mathrm{XPD}_{\min }$ is $27.12 \mathrm{~dB}$, almost $6 \mathrm{~dB}$ below the specification of $33 \mathrm{~dB}$.

In the final stage, 39291 variables are optimized at the same time. The final optimized layout complies with both, copolar and cross-polarization requirements at the three frequencies. Fig. 4 shows the evolution of the copolar and crosspolar components for Y polarization at $13.20 \mathrm{GHz}$ for the three stages. This polarization and frequency represent the worst case in the optimization process. For the final layout, the minimum value of $\mathrm{XPD}_{\min }$ in the $10 \%$ bandwidth is $33 \mathrm{~dB}$ in zone 1 for polarization $\mathrm{Y}$ at $13.20 \mathrm{GHz}$. Compared to the worst case of the initial design in stage 1 , the $\mathrm{XPD}_{\min }$ has improved $8.6 \mathrm{~dB}$.

\section{CONCLUSION}

In this work, a multi-frequency optimization procedure based on the generalized intersection approach and a multiresonant unit cell has been presented. The design process is divided into three stages. First, a initial design at central frequency is carried out. This first layout complies with the copolar requirement at central frequency, but it is narrowband and cross-polarization requirements were not taken into account. Then, a broadband optimization using two degrees of freedom (DoF) is carried out, imposing requirements in the minimum copolar gain and minimum crosspolar discrimination. The result of this second stage is a reflectarray that is close to fulfil specifications in the copolar pattern in the whole band. Finally, for the third stage the number of DoF per element is increased to six and the final optimized layout complies with both, copolar and crosspolarization requirements. This procedure was applied to a very large reflectarray for direct-to-home application with southern Asian coverage to work in a $10 \%$ bandwidth with improved cross-polarization performance, obtaining excellent results.

\section{ACKNOWLEDGMENT}

The authors would like to thank Dr. R. Florencio, Prof. R. R. Boix and Prof. J. A. Encinar for providing the MoM-LP software for the analysis of the reflectarray cell.

\section{REFERENCES}

[1] J. Huang and J. A. Encinar, Reflectarray Antennas. Hoboken, NJ, USA: John Wiley \& Sons, 2008.

[2] D. M. Pozar, "Wideband reflectarrays using artificial impedance surfaces," Electron. Lett., vol. 43, no. 3, pp. 148-149, Feb. 2007.

[3] E. Carrasco, J. A. Encinar, and M. Barba, "Bandwidth improvement in large reflectarrays by using true-time delay," IEEE Trans. Antennas Propag., vol. 56, no. 8, pp. 2496-2503, Aug. 2008. 


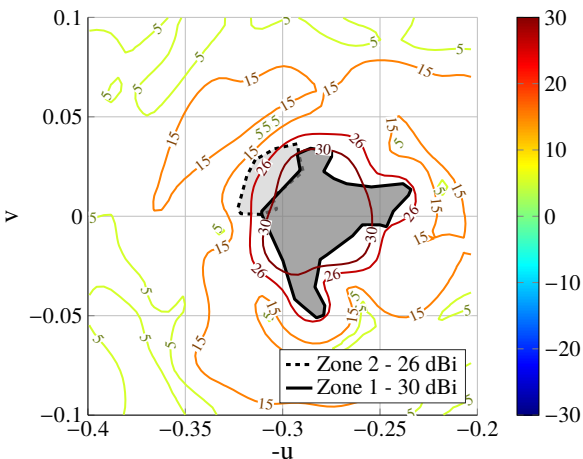

(a)

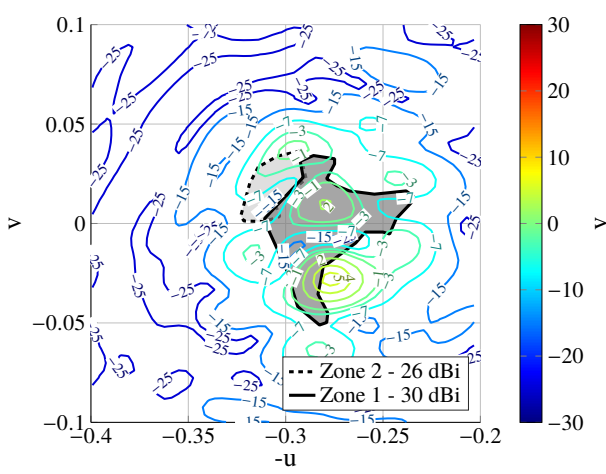

(d)

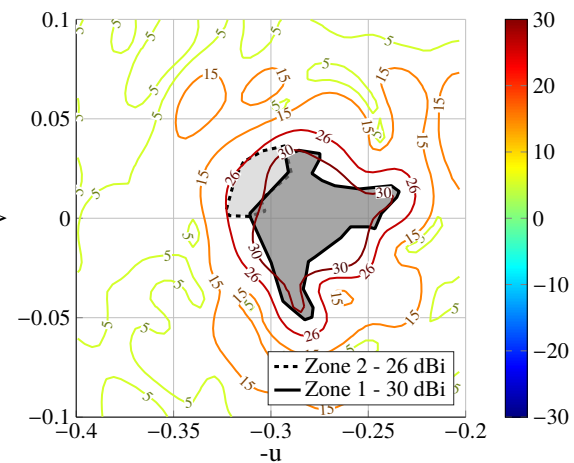

(b)

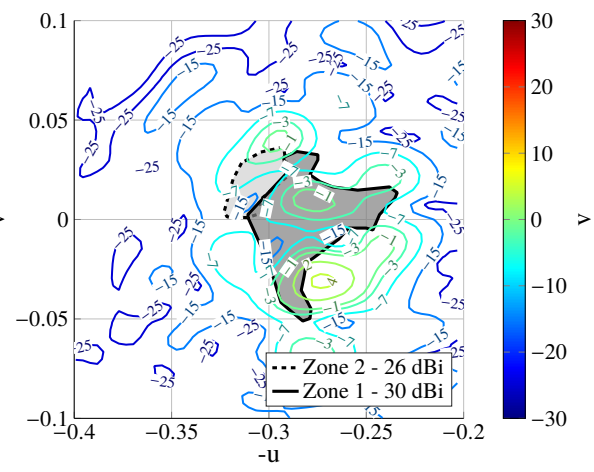

(e)

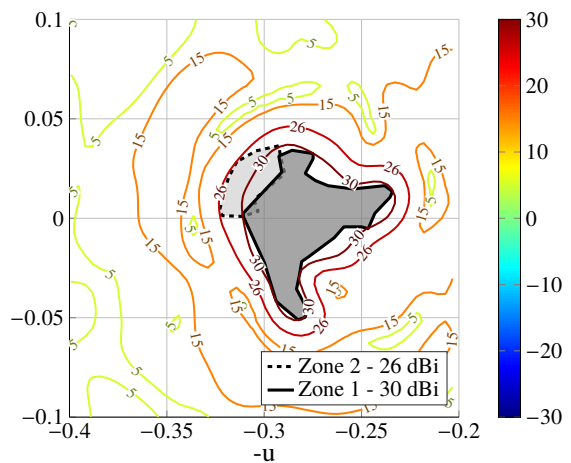

(c)

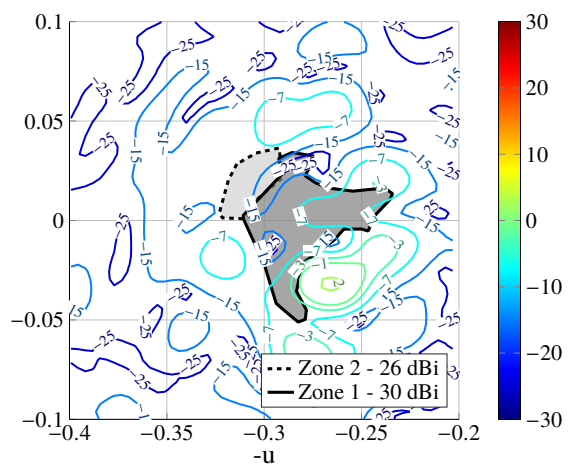

(f)

Fig. 4. Copolar (top) and crosspolar (bottom) radiation patterns for Y polarization (all of them in $\mathrm{dBi}$ ) at $13.20 \mathrm{GHz}$ for (a), (d) stage 1; (b), (e) stage 2; and (c), (f) stage 3 of the broadband design procedure.

[4] D. R. Prado, M. Arrebola, M. R. Pino, R. Florencio, R. R. Boix, J. A. Encinar, and F. Las-Heras, "Efficient crosspolar optimization of shaped-beam dual-polarized reflectarrays using full-wave analysis for the antenna element characterization," IEEE Trans. Antennas Propag., vol. 65, no. 2, pp. 623-635, Feb. 2017.

[5] R. Florencio, R. R. Boix, and J. A. Encinar, "Enhanced MoM analysis of the scattering by periodic strip gratings in multilayered substrates," IEEE Trans. Antennas Propag., vol. 61, no. 10, pp. 5088-5099, Oct. 2013.

[6] J. A. Encinar, R. Florencio, M. Arrebola, M. A. Salas-Natera, M. Barba, J. E. Page, R. R. Boix, and G. Toso, "Dual-polarization reflectarray in Ku-band based on two layers of dipole arrays for a transmit-receive satellite antenna with South American coverage," Int. J. Microw. Wirel. Technol., vol. 10, no. 2, pp. 149-159, 2018.

[7] "SES-12's mission," https://www.ses.com/our-coverage/satellites/365 [Accessed: 23 September 2019].

[8] D. R. Prado, M. Arrebola, M. R. Pino, and F. Las-Heras, "Improved reflectarray phase-only synthesis using the generalized intersection approach with dielectric frame and first principle of equivalence," Int. J. Antennas Propag., vol. 2017, pp. 1-11, May 2017.

[9] D. R. Prado, J. A. López-Fernández, M. Arrebola, M. R. Pino, and G. Goussetis, "General framework for the efficient optimization of reflectarray antennas for contoured beam space applications," IEEE Access, vol. 6, pp. 72 295-72310, 2018. 